
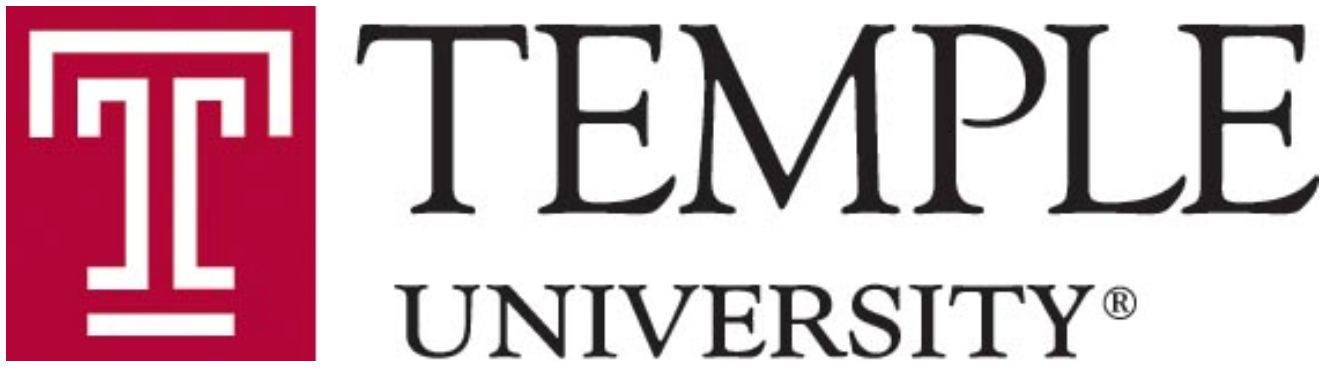

\title{
Ambiguity, Social Opinion and the Use of Common Property Resources
}

\author{
by \\ Dimitrios Diamantaras \\ Robert P. Gilles \\ Department of Economics \\ DETU Working Paper 10-06
}

April 2010

1301 Cecil B. Moore Avenue, Philadelphia, PA 19122 http://www.temple.edu/cla/economics/ 


\title{
Ambiguity, Social Opinion and the Use of Common Property Resources*
}

\author{
Dimitrios Diamantaras $^{\dagger} \quad$ Robert P. Gilles ${ }^{\ddagger}$
}

July 2008

Revised: December 2009

\begin{abstract}
In this paper we argue that ambiguity, combined with social opinion formation can be represented as part of a game-theoretic equilibrium concept that transcends the standard Nash equilibrium concept, applied to a model of the tragedy of the commons. Our modeling can shed some light on the international environment crisis and the relevant ongoing international negotiations. We conclude that social opinion formation in most cases has a significant impact on equilibrium common property resource usage.
\end{abstract}

${ }^{*}$ We thank Cees Withagen for an enlightening discussion with one of the authors about this work and participants in the 2008 International Meetings of the Social Choice and Welfare Society in Montreal for their useful questions. We are very grateful for the remarks and suggestions by three referees that allowed us to improve this paper.

†Department of Economics, Temple University, Philadelphia, PA. Email: dimitris@temple.edu.

*Management School, Queen's University, 25 University Square, Belfast BT7 1NN, UK. Email: r.gilles@qub.ac.uk Financial support from the Netherlands Organization for Scientific Research (NWO) is gratefully acknowledged. 


\section{Ambiguity, the environment and social opinion formation}

The current climate debate and the negotiations over global emission reductions bring up multiple pressing questions for economists and game theorists on the issue of using common property resources. Economists can contribute to the public debate by clarifying the incentives of decision makers. In this paper we discuss an innovative approach for understanding the influence of public discourse on individual incentives and behavior. Decision makers, being members of certain populations of economic agents, are usually strongly influenced by public opinion formation regarding global warming and the effects of emission abatement on environment and the economy. This, in turn, affects the decision making processes concerning the usage of common resources. In particular, it affects global pollution abatement policy formation.

More specifically, we take a step towards the development of a tractable model of the influence of public discourse on equilibrium behavior in a common resource game. Our approach takes us away from standard game theoretic equilibrium concepts such as Nash and Bayesian equilibrium. Instead we focus on the direct incorporation of ambiguity and social influence on game theoretic decision processes. In particular, we show that certain social influences - public opinion formation in particular-might guide decision makers to a more efficient equilibrium state than standard concepts would support.

We use the standard common property resource or tragedy of the commons game as a vehicle to study the incentives involved in global emission reductions. The tragedy of the commons acts in many respects as a metaphor for the relevant issues. In this normal form game there is a stark difference between the socially optimal or Pareto efficient state and the standard Nash equilibrium state in which players act only in their self-interest. This fundamental problem has already been addressed extensively in the literature on environmental economics.

In that literature, authors have examined a linear model of common property resources management, with emphasis on self-enforcing and stable international environmental agreements (Barrett, 2003; Ulph, 2004; Kolstad, 2007). These contributions study coalition formation and dynamic negotiation processes, incorporate uncertainty with respect to the marginal cost of the use of the commons, and introduce dynamic accumulation of pollution stock. However, they use a restricted set of game theoretic equilibrium concepts, such as Nash equilibrium and subgame perfection.

A drawback of these standard equilibrium concepts is that all of these equilibrium concepts assume that individuals evaluate outcomes by means of Subjective Expected Utility 
(SEU) theory (Savage, 1954). Well-established experimental evidence urges the consideration of departures from expected utility. The evidence presented by, e.g., Ellsberg (1961) and Camerer and Weber (1992) questions whether standard probabilities can capture the nature of individual beliefs. Further, Eichberger, Kelsey, and Schipper (2006) discuss an experiment in which subjects experience ambiguity due to the identity of the other player in a two-player game, who could be sophisticated or not; when facing the unsophisticated player, subjects felt more ambiguous and played more conservatively.

A major alternative to SEU is the theory of decision making under ambiguity (Schmeidler, 1989). The basic idea dates back to the work of Knight (1921) on uncertainty: individuals cannot know precisely the probabilities of all payoff-relevant events. Combining this with the mathematical theory of capacities ${ }^{1}$ originating from Choquet (1954) resulted in Choquet Expected Utility (CEU) theory. This theory postulates that individuals maximize an expected utility where the expectation is taken using a capacity instead of a probability distribution. A capacity can be used to describe the degree to which the individual's beliefs are ambiguous. The farther below unity the sum of all capacities is from unity, the more ambiguous the belief system these capacities represent.

Founded on CEU, the ambiguity equilibrium concept of Eichberger and Kelsey (2000) targets the analysis of games of complete information played by players who are affected by ambiguity in their beliefs. The presence of ambiguity implies that a player is not confident about his or her subjective probability assignment to the various states of the world that may arise. Thus, these players maximize an expected payoff based on a capacity rather than on a probability distribution to represent the probabilistic evaluation of the actions of others made by each player. Recently, a particular variation of the ambiguity equilibrium concept-based on so-called neo-additive capacities - has been developed in Eichberger and Kelsey (2006), Chateauneuf, Eichberger, and Grant (2007), Eichberger, Kelsey, and Schipper (2007), and Eichberger and Kelsey (2007). Ambiguity equilibrium under neo-additive capacities is arguably the proper solution concept to apply to the problem of common resource usage.

Chateauneuf, Eichberger, and Grant (2007) showed that for neo-additive capacities, the ambiguity equilibrium concept obtains a very tractable and intuitive formulation. Players simply weigh three terms in their payoff functions. The first term represents their most optimistic assessment of what the others will play, the second the most pessimistic assessment of what the others will play, and the third their standard expected utility payoff. The weight with which the first term is considered is called the "degree of optimism", and the weight of the second term the "degree of pessimism". This formulation calls upon the implementation of a

\footnotetext{
${ }^{1}$ Capacities are probabilities that can sum up to less than unity.
} 
"neo-additive" payoff function.

We consider two implementations of the neo-additive reformulation of the commons game using the standard ambiguity formulation introduced by Chateauneuf, Eichberger, and Grant (2007). Using this standard implementation in the commons game, optimistic - respectively pessimistic - attitudes are formulated through the global maximum payoff-respectively the global minimum payoff. In this regard, both attitudes reflect maximal antagonism among the participants in the commons game. We investigate two possible cases.

First, if both players have the same degrees of optimism and pessimism, social opinion formation can, in principle, guide the equilibrium outcome to a Pareto optimal state. This requires finding a delicate balance between optimism and pessimism. If these degrees of optimism and pessimism deviate from this equilibrium, suboptimal extraction from the commons results.

Second, if the players are so asymmetric that one is optimistic and the other pessimistic, then there is always overuse of the commons; any increase in optimism moves the equilibrium further away from the Pareto optimal state, and any increase in pessimism moves the equilibrium closer to it.

We believe that the standard formulation of Chateauneuf, Eichberger, and Grant (2007) does not completely capture the current state of affairs in the global economy regarding the pollution abatement situation. Rather, countries as the main decision makers are structurally positioned in the extraction from the global commons. One can clearly distinguish "leaders" from "followers". This informs our investigation how structural or positional causes for ambiguity affect decision making of the players in our simple commons game.

Thus, we consider a modified implementation of players' attitudes that reflect ambiguity about the structural positions of the different players in the decision-making processes. In particular, optimism corresponds to the player's belief that she has a position of leadership as a first mover in the game. Similarly, pessimism now reflects a player's belief that he has a follower role. As such, this case corresponds to a more practical attitude towards pessimism and optimism in the context of the commons game.

We emphasize that these optimistic and pessimistic beliefs are (implicitly) based upon social opinion formation, which in turn is founded on the standard Stackelberg model of leadership in duopolistic market games. ${ }^{2}$ Our main insight is that under such a leader-follower formulation of ambiguity there results greater overuse of the commons relative to the Pareto

\footnotetext{
${ }^{2}$ We use here the fact that the tragedy of the commons has fully the same functionality as a standard duopolistic market.
} 
efficient level, as compared to the standard ambiguity equilibrium. Moreover, increased optimism of a player's leadership role increases the usage of the commons. Finally, a higher degree of pessimism about a player's follower role results unambiguously in lower levels of inefficiency through the decrease of the overuse of the commons. Our main conclusion is that leadership positions have a harmful effect on the pollution abatement process and that lower levels of ambiguity about such leadership positions improve the efficient use of common pool resources.

It is this last result that points to the potential value of this game theoretic analysis of the commons game. It shows that if public opinion formation results in the leadership-based concept of the most selfish move of the stronger player, then the commons is overused even more. Furthermore, the more pessimistic the weaker player is, the less the commons is used.

In the context of our results, it would appear that developing countries have been acting as followers over a long stretch of history, but have relatively recently switched their attitude to a more extreme, and optimistic, one, closer to the behavior of the players in the standard ambiguity equilibrium. The results from our analysis then would be broadly in agreement with the outcomes we have observed in terms of atmospheric pollution over the past several decades.

The remainder of this paper is organized as follows. Section 2 introduces the game theoretic tools and equilibrium concepts. We consider the tragedy of the commons game and determine its equilibria for the various implementations of ambiguity. In Section 3 we derive the equilibria of the commons game under structural ambiguity. Section 4 concludes. 


\section{Ambiguity and the use of common resources}

We consider non-cooperative games in normal form with two players, denoted by 1,2 or generically by $i$ and $-i$. For each player $i$ we denote by $S_{i}$ player $i$ 's strategy set and define $S=S_{1} \times S_{2}$ as the resulting set of strategy tuples. Finally, for each player $i$ we introduce a payoff function $\pi_{i}: S \rightarrow \mathbb{R}$. The game can now be represented as a pair $(S, \pi)$, where $\pi=\left(\pi_{1}, \pi_{2}\right): S \rightarrow \mathbb{R}^{2}$.

Considering decision-making processes related to the use of common resources, it is natural to take into account how the players perceive the actions of each other. ${ }^{3}$ This refers to the ambiguity in each player's mind about how other players might come to decisions. This ambiguity can be captured by an appropriately constructed equilibrium notion, the ambiguity equilibrium concept. In this section we follow Eichberger, Kelsey, and Schipper (2007), who build upon Eichberger and Kelsey (2000). We introduce ambiguity through four elements for each player $i$ who participates in a game. For player $i$ this concerns the quadruplet $\left(M_{i}, \lambda_{i} ; m_{i}, \gamma_{i}\right)$ representing the following elements.

Optimistic beliefs. Each player $i$ formulates well-defined optimistic expectations with regard to her payoffs in the game. These expectations describe the best that can occur in the game concerning this player. The optimistic payoff function of player $i$ is the function $M_{i}: S_{i} \rightarrow \mathbb{R}$ assigning to every strategy $x_{i} \in S_{i}$ of player $i$ its maximally expected payoff $M_{i}\left(x_{i}\right) \in \mathbb{R}$ defined by

$$
M_{i}\left(x_{i}\right)=\max _{x_{-i} \in S_{-i}} \pi_{i}\left(x_{i}, x_{-i}\right)
$$

The number $\lambda_{i} \in[0,1]$ represents the weight that player $i$ puts on her optimistic beliefs, in other words, the degree of optimism of player $i$. If $\lambda_{i}=0$, player $i$ has no expectation that she will receive maximal payoffs in the game, while $\lambda_{i}=1$ refers to the other extreme case that player $i$ is fully convinced that she will only receive maximal payoffs.

Pessimistic beliefs. Similarly, each player $i$ formulates pessimistic expectations with regard to her payoffs in the game. These expectations describe the worst that this player can imagine happening to her in the game. The pessimistic payoff function of player $i$ is the function $m_{i}: S_{i} \rightarrow \mathbb{R}$ assigning to every strategy $x_{i} \in S_{i}$ of player $i$ the minimally

\footnotetext{
${ }^{3}$ We remark here that the main application of our model is the abatement of pollution in the global economy. Players stand for countries that pollute the global environment through their productive activities.
} 
expected payoff $m_{i}\left(x_{i}\right) \in \mathbb{R}$ defined by

$$
m_{i}\left(x_{i}\right)=\min _{x_{-i} \in S_{-i}} \pi_{i}\left(x_{i}, x_{-i}\right)
$$

The number $\gamma_{i} \in[0,1]$ represents the weight that player $i$ puts on her pessimistic beliefs, in other words, player $i$ 's degree of pessimism. If $\gamma_{i}=0$, player $i$ has no expectation that she will receive minimal payoffs in the game, while $\gamma_{i}=1$ refers to the other extreme case that player $i$ is fully convinced that she will only receive minimal payoffs.

The belief-system $\left(\lambda_{i}, M_{i} ; \gamma_{i}, m_{i}\right)_{i=1,2}$ is proper if for every player $i$ it holds that $\lambda_{i}+\gamma_{i} \leqslant 1$, where $\lambda_{i}+\gamma_{i}$ is the degree of ambiguity of player $i$. This allows us to introduce the equilibrium concept that underlies the rest of our analysis:

Definition 2.1 A strategy tuple $x^{\star} \in S$ is an ambiguity equilibrium in the game $(S, \pi)$ for the proper belief system $\left(\lambda_{i}, M_{i} ; \gamma_{i}, m_{i}\right)_{i=1,2}$ if $x^{\star}$ is a Nash equilibrium in the modified game $(S, \bar{\pi})$, where $\bar{\pi}_{i}: S \rightarrow \mathbb{R}$ for each player $i$ is a modified payoff function given by

$$
\bar{\pi}_{i}\left(x_{i}, x_{-i}\right)=\lambda_{i} M_{i}\left(x_{i}\right)+\gamma_{i} m_{i}\left(x_{i}\right)+\left(1-\gamma_{i}-\lambda_{i}\right) \pi_{i}\left(x_{i}, x_{-i}\right)
$$

If the degree of ambiguity is zero, the modified payoff formulation (1) reduces to the standard payoff function. Chateauneuf, Eichberger, and Grant (2007) provide an axiomatic foundation for the equilibrium under ambiguity that underlies the ambiguity equilibrium we use here. ${ }^{4}$

\subsection{A commons game}

Our goal is to prepare the ground for an application of the ambiguity equilibrium concept, rather than to refine its theory, and to introduce a formalization of the effects of social opinions on the outcome of the interaction between nations in the global community. We explore this in a model that is sufficiently simple and compelling as to have a hope of practical application in the field of environmental economics, and, more generally, in public economics.

\footnotetext{
${ }^{4}$ As pointed out in the papers by Eichberger and Kelsey, the modified payoff function (1) is in fact the Choquet integral (Choquet, 1954) of the payoff function for a neo-additive capacity based on the ambiguity represented in the belief system $\left(\lambda_{i}, M_{i} ; \gamma_{i}, m_{i}\right)_{i=1,2}$. Proposition 3.1 in Eichberger, Kelsey, and Schipper (2007) states that for every pure strategy Nash equilibrium of the modified game there is a pure strategy equilibrium under ambiguity in which each player $i$ has degree of optimism $\lambda_{i}$ and of pessimism $\gamma_{i}$. Eichberger, Kelsey, and Schipper (2007), Eichberger and Kelsey (2000), Eichberger and Kelsey (2006) are good introductions to the theory of equilibrium under ambiguity. We also note here that the definition of equilibrium under ambiguity for more than two players is more subtle than a direct extension of the one just given. We refer to Eichberger and Kelsey (2000) for a discussion of the subtleties involved. We only consider two-player games in this paper and leave extensions to more players for future work.
} 
We interpret the players to explicitly stand in for certain nations. Moreover, we explicitly assume that within these nations there is a public debate about policy goals regarding the strategic extraction of the common pool resources introduced through this game.

We employ the ambiguity equilibrium concept to flesh out asymmetry in the degrees of (national) optimism and pessimism. The asymmetry is intended to capture in our simple model differing world views, such as a very optimistic view of the effects of global warming, as generally seen in past behavior of the US in international negotiations, and a pessimistic view as seen almost everywhere else. Admittedly, this may appear to be an oversimplification, but the study of international responses to environmental change deals with such complex issues we feel a simple approach is best, as long as it captures a relevant aspect of the gametheoretic interaction. The emphasis we place on simplicity in modelling is shared by some prominent environmental economists — see, for instance, Barrett (2003).

We investigate a version of the tragedy of the commons, adapted from Falk, Fehr, and Fischbacher (2001). ${ }^{5}$ The commons game is formally represented as a two-player normal form game $\Gamma=\left(X^{2}, \pi\right)$, where $X=[0, a], a>0$, is each player's action set and $\pi_{i}: X^{2} \rightarrow \mathbb{R}$ is player $i$ 's $(i=1,2)$ payoff function. Each player $i \in\{1,2\}$ selects an activity level $x_{i} \in X=$ $[0, a]$. The payoff of each player $i$ is given by

$$
\pi_{i}\left(x_{i}, x_{-i}\right)=a x_{i}-x_{i}\left(x_{i}+x_{-i}\right)
$$

The parameter $a>0$ describes the maximal extraction from the commons. This upper bound $a$ is exactly the value which results in a zero payoff even if the other player is inactive. The lower bound is that of inactivity. ${ }^{6}$

The Nash equilibrium activity levels $x_{1}^{*}, x_{2}^{*}$ can be computed as

$$
x_{1}^{*}=x_{2}^{*}=\frac{a}{3}=x^{*}
$$

The resulting Nash equilibrium payoff level for each player is

$$
\pi_{1}^{*}=\pi_{2}^{*}=\frac{a^{2}}{9}
$$

The socially optimal state is described as the maximizers of the sum of payoffs. For reference,

\footnotetext{
${ }^{5}$ This version of the commons game is quadratic. Ulph (2004) and related work apply a linear formulation of this game. The quadratic version seems more appropriate to reflect a true common resources situation in which extraction more directly affects the benefits of the other players.

${ }^{6}$ The original formulation in Falk, Fehr, and Fischbacher (2001) uses a second parameter $b>0$ with $\pi_{i}(x)=$ $a x_{i}-b x_{i} \sum_{j=1}^{n} x_{j}$. We have rescaled units by selecting $b=1$.
} 
the socially optimal activity level of each player is determined as

$$
\hat{x}_{1}=\hat{x}_{2}=\frac{a}{4}=\hat{x}
$$

which results in the optimal payoffs

$$
\hat{\pi}_{1}=\hat{\pi}_{2}=\frac{a^{2}}{8}
$$

We use the standard Nash equilibrium and the social optimum as benchmarks in our discussion of various alternative equilibrium concepts.

\subsection{Ambiguity equilibrium}

We consider the standard ambiguity equilibrium concept for the commons game formulated in the previous section founded on neo-additive capacities expressing such ambiguity. It is easy to compute that the optimistic payoff functions are given by

$$
M_{i}\left(x_{i}\right)=\pi_{i}\left(x_{i}, 0\right)=a x_{i}-x_{i}^{2} .
$$

and that the pessimistic payoff functions are

$$
m_{i}\left(x_{i}\right)=\pi_{i}\left(x_{i}, a\right)=-x_{i}^{2} .
$$

We consider ambiguity equilibria for two different sets of degrees of optimism and pessimism: the case of symmetric degrees, i.e., $\lambda_{1}=\lambda_{2}=\lambda$ and $\gamma_{1}=\gamma_{2}=\gamma$, and the antipodal case in which one player is optimistic and the other player is pessimistic.

\section{Symmetric ambiguity}

Following the formal definitions in the previous section, the symmetric ambiguity equilibria can now be formulated as the Nash equilibria for the modified commons game $\Gamma^{s}(\lambda, \gamma)$ with payoff functions given by

$$
\begin{aligned}
U_{i}\left(x_{i}, x_{-i}\right) & =\lambda M_{i}\left(x_{i}\right)+\gamma m_{i}\left(x_{i}\right)+(1-\lambda-\gamma) \pi_{i}\left(x_{i}, x_{-i}\right) \\
& =\lambda\left(a x_{i}-x_{i}^{2}\right)+\gamma\left(-x_{i}^{2}\right)+(1-\lambda-\gamma)\left[a x_{i}-x_{i}\left(x_{i}+x_{-i}\right)\right] \\
& =(1-\gamma) a x_{i}-x_{i}^{2}-(1-\lambda-\gamma) x_{i} x_{-i} .
\end{aligned}
$$




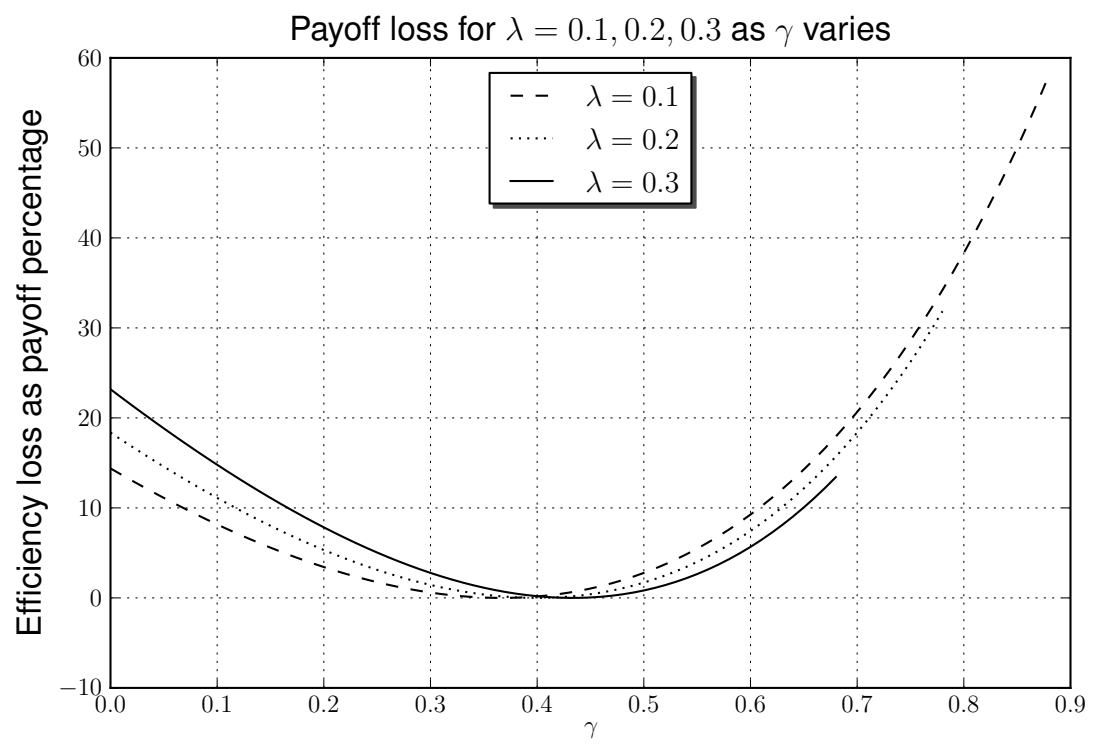

Figure 1: Representative percentage payoff losses in the symmetric case for $a=1$.

We compute from (9) for $i=1,2$ that each player's first order condition for the symmetric ambiguity equilibrium is given by

$$
\frac{\partial U_{i}}{\partial x_{i}}\left(x_{i}, x_{-i}\right)=(1-\gamma) a-2 x_{i}-(1-\lambda-\gamma) x_{-i}=0 .
$$

This results in a unique equilibrium under symmetric ambiguity given by

$$
x_{i}^{s}=\frac{(1+\lambda+\gamma)(1-\gamma) a}{4-(1-\lambda-\gamma)^{2}}, \quad i=1,2 .
$$

The resulting payoffs for this equilibrium under symmetric ambiguity is thus derived as

$$
U_{i}^{s}=a^{2}(1+\lambda+\gamma)(1-\gamma) \frac{1-\lambda^{2}+\gamma^{2}+2 \gamma}{\left[4-(1-\lambda-\gamma)^{2}\right]^{2}} .
$$

A numerical analysis of these equilibrium payoffs in comparison with the socially optimal payoff level results in the graphs depicted in Figure 1. This representation depicts the difference of the Pareto optimal and the equilibrium payoffs as a percentage of the Pareto optimal payoff level.

We note that the efficiency losses from symmetric ambiguity can be significant. However, the analysis also shows that for every degree of optimism there corresponds a certain degree of pessimism such that the resulting equilibrium is fully optimal. This implies that under 
symmetric ambiguity, public discussion can, in principle, guide us to an efficient state. In any case the efficiency losses can, at least, be limited by the appropriate guidance through social opinion formation.

Our analysis can be summarized as follows:

Theorem 2.2 Under symmetric ambiguity, the following statements hold:

(i) For every degree of optimism $0 \leqslant \lambda \leqslant \frac{1}{2}$ there exists a unique degree of pessimism $\gamma^{*}(\lambda)$ such that $\lambda+\gamma^{*}(\lambda) \leqslant 1$ and the corresponding symmetric ambiguity equilibrium results in a Pareto optimal extraction from the commons, that is, $x^{s}=\hat{x}$.

(ii) For every degree of pessimism $0 \leqslant \gamma<\frac{1}{2}$ there exists a threshold value $\lambda_{\gamma}$ of the degree of optimism such that

(a) above that threshold value $\lambda_{\gamma}$ an increase in the degree of optimism $\lambda>\lambda_{\gamma}$ results in an increased overuse of the commons in the corresponding symmetric ambiguity equilibrium, and

(b) below that threshold value $\lambda_{\gamma}$ a decrease in the degree of optimism $\lambda<\lambda_{\gamma}$ results in an increased underuse of the commons in the corresponding symmetric ambiguity equilibrium.

In the context of the statements in the theorem, we point out the various limit cases of complete or "unbridled" optimism and pessimism. First, under unbridled optimism represented by $\lambda=1$ and $\gamma=0$, social opinion formation results in an equilibrium with

$$
x_{i}^{s}=\frac{a}{2} \quad \text { and } \quad U_{i}^{s}=0
$$

On the other hand, unbridled pessimism is represented by $\lambda=0$ and $\gamma=1$ resulting in an equilibrium with

$$
x_{i}^{s}=0 \quad \text { and } \quad U_{i}^{s}=0 .
$$

This case is equally disastrous; both unbridled social optimism and pessimism leads to complete depletion of payoffs.

The modelling of a player who is simultaneously optimistic and pessimistic in a game with a one-dimensional strategy set, such as the game we consider here, may be considered far-fetched. How can the player be optimistic and pessimistic at the same time? If the set over which the ambiguity is defined is multi-dimensional, we could imagine that the player 
is optimistic over some dimensions and pessimistic over others; this is allowed under the Choquet integral formulation of beliefs under ambiguity. In this paper, the set over which ambiguity applies is one-dimensional. Intuitively, the results of this section are easier to consider when we allow only $\lambda$ or only $\gamma$ to differ from zero. In the analysis presented in the next subsection we only allow such situations.

\section{Antipodal ambiguity}

Under antipodal ambiguity we assume that one player is optimistic and the other player is pessimistic, although we keep the possibility of having various degrees of optimism and pessimism. Without loss of generality we suppose that player 1 is the optimist and player 2 is the pessimist. This case corresponds to the hypothesis that $\lambda_{1}=\lambda>0$ and $\gamma_{1}=0$, while $\lambda_{2}=0$ and $\gamma_{2}=\gamma>0$. This results in an antipodal ambiguity equilibrium being a Nash equilibrium of the modified commons game $\Gamma^{a}(\lambda, \gamma)$ with payoff functions given by

$$
\begin{aligned}
V_{1}\left(x_{1}, x_{2}\right) & =\lambda M_{1}\left(x_{1}\right)+(1-\lambda) \pi_{1}\left(x_{1}, x_{2}\right) \\
& =\lambda\left(a x_{1}-x_{1}^{2}\right)+(1-\lambda)\left(a x_{1}-x_{1}\left(x_{1}+x_{2}\right)\right) \\
& =a x_{1}-x_{1}^{2}-(1-\lambda) x_{1} x_{2},
\end{aligned}
$$

and

$$
\begin{aligned}
V_{2}\left(x_{1}, x_{2}\right) & =\gamma m_{2}\left(x_{2}\right)+(1-\gamma) \pi_{2}\left(x_{1}, x_{2}\right) \\
& =\gamma\left(-x_{2}^{2}\right)+(1-\gamma)\left(a x_{2}-x_{2}\left(x_{1}+x_{2}\right)\right) \\
& =(1-\gamma)\left(a x_{2}-x_{1} x_{2}\right)-x_{2}^{2} .
\end{aligned}
$$

We can now easily compute the first order conditions for equilibrium:

$$
\begin{aligned}
& \frac{\partial V_{1}}{\partial x_{1}}=a-2 x_{1}-(1-\lambda) x_{2}=0, \\
& \frac{\partial V_{2}}{\partial x_{2}}=(1-\gamma)\left(a-x_{1}\right)-2 x_{2}=0 .
\end{aligned}
$$

This results in a unique antipodal ambiguity equilibrium given by

$$
\begin{aligned}
& x_{1}^{a}=\frac{1+\lambda+\gamma-\lambda \gamma}{4-(1-\lambda)(1-\gamma)} a, \\
& x_{2}^{a}=\frac{1-\gamma}{4-(1-\lambda)(1-\gamma)} a .
\end{aligned}
$$


Tedious computation confirms that the optimistic player 1's activity level is more than the efficient level of $a / 4$, while the pessimistic player 2's activity level is less if $\gamma$ is large enough. However, the sum $x_{1}^{a}+x_{2}^{a}$ is unambiguously more than the efficient level of $a / 2$, so we have overuse of the commons.

The comparative statics now are clear-cut. We find the following.

$$
\frac{\partial x_{1}^{a}}{\partial \lambda}=\frac{2 a(1-\gamma)}{[4-(1-\lambda)(1-\gamma)]^{2}}>0
$$

The interpretation of this result is that as the optimistically biased player becomes more optimistic, her activity level increases. This is consistent with the general insight from the literature that optimism leads away from efficient choices in situations of the provision of a public good, although we do not know of any previous analysis that has shown this in an asymmetric model like ours.

Similarly, we derive that

$$
\frac{\partial x_{2}^{a}}{\partial \lambda}=-a \frac{(1-\gamma)^{2}}{[4-(1-\lambda)(1-\gamma)]^{2}}<0 .
$$

This implies that the pessimistically biased player reacts to the increase in optimism of the other player by reducing its harmful activity.

We can also see what the overall effect on the activity will be by a change in $\lambda$. This is simply the sum of the two partial derivatives we have just calculated, and it equals

$$
\frac{\partial x_{1}^{a}}{\partial \lambda}+\frac{\partial x_{2}^{a}}{\partial \lambda}=a \frac{(1-\gamma)[2-(1-\gamma)]}{[4-(1-\lambda)(1-\gamma)]^{2}}>0
$$

We see that the increase in the optimist's activity level clearly over-compensates the decrease of the pessimist's activity.

We also look at the effect of an increase in the degree of pessimism $\gamma$ of the pessimistically biased player. We derive that

$$
\frac{\partial x_{1}^{a}}{\partial \gamma}=\frac{2 a(1-\lambda)}{[4-(1-\lambda)(1-\gamma)]^{2}}>0 .
$$

Hence, if the pessimist becomes more so, the optimist takes advantage by increasing its own activity level. The effect on the pessimist's own activity level is

$$
\frac{\partial x_{2}^{a}}{\partial \gamma}=-a \frac{4}{[4-(1-\lambda)(1-\gamma)]^{2}}<0 .
$$


We see that the pessimist reduces activity level drastically in response to an increase in its pessimism. In fact, it is also true that the total activity level is reduced:

$$
\frac{\partial x_{1}^{a}}{\partial \gamma}+\frac{\partial x_{2}^{a}}{\partial \gamma}=a \frac{2(1-\lambda)-4}{[4-(1-\lambda)(1-\gamma)]^{2}}<0
$$

We summarize our results in this subsection in the following.

Theorem 2.3 Consider the tragedy of the commons under antipodal ambiguity with one optimistically biased player, the "optimist", and one pessimistically biased player, the "pessimist”. Then the following statements hold:

(i) There is always overuse in the resulting antipodal ambiguity equilibrium.

(ii) An increase in the degree of optimism of the optimistic player increases that player's activity level, reduces the pessimist's activity level, and increases the sum of their activity levels.

(iii) Finally, an increase in the pessimist's degree of pessimism increases the optimist's activity level, decreases the pessimist's activity level, and decreases the sum of their activity levels. 


\section{Structural Ambiguity}

In this section we inject a more nuanced implementation of optimism and pessimism into the reasoning of the decision makers about each other. Inspired by the ambiguity equilibrium formulation, we impose modified payoff functions for the two decision makers that reflect their respective view on their structural position in the pollution abatement negotiations. This form of ambiguity, thus, concerns whether a player has a leadership position or a following position within the global pollution situation. We first debate the foundations for this form of ambiguity and subsequently develop a formal model to express these concerns.

\section{Leadership and Social Opinion Formation}

We imagine the players as influenced by public perceptions within their countries as to their relative strength in the underlying global commons game. We intentionally allow only one aspect of these extractions from the commons into our enhanced model; namely the structural ambiguity regarding their leadership or follower position in the global community that affects the various nations.

Given the contemporary global political situation it is natural to assume that certain countries have a rather different perception of the global commons problem than others. Here we simplify this by contrasting large, influential economies from developing, minor economies. Large, developed economies are in a position of leadership, while minor economies are in a position of following the announcements of these leaders. In the simple two-player commons game we now assume that up to a certain degree player 1 is a potential leader, while player 2 is a potential follower in the negotiations about the use of the commons. Consequentially, the leader is assumed to be optimistic in her perception of the game, while the follower is pessimistic in that regard. This is in particular founded on the perception that leadership implies some form of control of the decision-making processes.

Thus, the ambiguity of the players about their respective actions and choices in this game is replaced by ambiguity about their structural positions in the game. Player 1 is optimistic about having a leadership position, while player 2 is pessimistic that it is positioned as a follower in the social structure of the decision-making processes about the use of the commons. We assume that both players still have different degrees of optimism (player 1) and pessimism (player 2) about their structural position. Hence, these two degrees still feature as parameters in our model. What is different from the preceding section is that the ambiguity regards being a leader or a follower and that this ambiguity comes from general perceptions in background populations of agents. We refrain from offering a detailed model of the transmission of opti- 
mistic and pessimistic opinions within large populations of agents for the same reason we do not model an explicit negotiation dynamic.

\section{A formal model}

To model the structural positions of the two players in the simple 2-player variation of the standard commons game, we invoke the Stackelberg leadership model of a sequential determination of the strategic values of $x_{i}, i=1,2$. Subsequently, informed by the ambiguity equilibrium concept, we introduce two ad hoc formulations of the leader's and follower's payoff functions. The leader's payoff is founded on the Stackelberg hypothesis that the follower always plays a best response, while the leader takes this response in full account. ${ }^{7}$ The leader's degree of optimism $\lambda>0$ is now simply her confidence that she actually will be in such a leadership position. In this regard, $\lambda$ is player 1's leadership degree.

The first step in our analysis is to determine the basic Stackelberg responses. Here, the follower's best response function derived from $\pi$ is now computed as

$$
\beta\left(x_{1}\right)=\frac{1}{2}\left(a-x_{1}\right)
$$

In the straightforward Stackelberg-leadership situation, the leader now maximizes her modified payoff function given by

$$
\pi_{1}\left(x_{1}, \beta\left(x_{1}\right)\right)=a x_{1}-x_{1}\left(x_{1}+\beta\left(x_{1}\right)\right)=\frac{a}{2} x_{1}-\frac{1}{2} x_{1}^{2} .
$$

This results in an optimum usage level for the leader of

$$
\sigma=\underset{x_{1}>0}{\arg \max } \pi_{1}\left(x_{1}, \beta\left(x_{1}\right)\right)=\frac{a}{2} .
$$

This allows us to formulate the corresponding payoff functions for the leader-follower structure as follows:

$$
W_{1}\left(x_{1}, x_{2}\right)=\lambda \pi_{1}\left(x_{1}, \beta\left(x_{1}\right)\right)+(1-\lambda) \pi_{1}\left(x_{1}, x_{2}\right) .
$$

Thus, player 1 - as a large economy - expects to be in a leadership position to a certain extend quantified through the degree $0<\lambda \leqslant 1$. In line with our previous discussion, $\lambda$ can be interpreted as the leadership degree for player 1.

\footnotetext{
${ }^{7}$ This is a consequence of the sequential structure of the decision making processes modelled through the subgame perfection of the equilibrium concept.
} 
We note that it is also possible to interpret $\sigma$ as the most selfish action that the optimistic player can take. In the previous section, this most extremely selfish action was the extreme one of appropriating the whole commons based on the ambiguity concept introduced in Eichberger, Kelsey, and Schipper (2007). Instead of this, here the beliefs of both players about the extremism of the most powerful player are moderated and we have chosen to moderate them by following the Stackelberg leader-follower analysis. Other ways to do this are certainly possible and might interface well with models of social belief formation. While this topic is interesting, it is beyond the scope of this paper.

Returning to the specification of payoffs, player 2-as the minor economy-expects to be in the position of a follower in relation to player 1 to an extent quantified by $0<\gamma \leqslant 1$. Here $\gamma$ can be denoted as player 2's follower degree. This results in the modified payoff function given by

$$
W_{2}\left(x_{1}, x_{2}\right)=\gamma \pi_{2}\left(\sigma, x_{2}\right)+(1-\gamma) \pi_{2}\left(x_{1}, x_{2}\right) \text {. }
$$

From the above we now compute that

$$
\begin{aligned}
& W_{1}\left(x_{1}, x_{2}\right)=\left(1-\frac{\lambda}{2}\right)\left(a x_{1}-x_{1}^{2}\right)-(1-\lambda) x_{1} x_{2}, \\
& W_{2}\left(x_{1}, x_{2}\right)=\left(1-\frac{\gamma}{2}\right) a x_{2}-x_{2}^{2}-(1-\gamma) x_{1} x_{2} .
\end{aligned}
$$

We now introduce the notion of a Leadership Equilibrium as a Nash equilibrium of the modified commons game $\Gamma^{\ell}(\lambda, \gamma)$, where the payoff functions of the two players are given by $W_{1}$, respectively $W_{2}$.

For the given parameters we derive the first order conditions necessary for this Leadership Equilibrium:

$$
\begin{aligned}
& \frac{\partial W_{1}}{\partial x_{1}}=\left(1-\frac{\lambda}{2}\right)\left(a-2 x_{1}\right)-(1-\lambda) x_{2}=0 \\
& \frac{\partial W_{2}}{\partial x_{2}}=\left(1-\frac{\gamma}{2}\right) a-2 x_{2}-(1-\gamma) x_{1}=0 .
\end{aligned}
$$

The Leadership Equilibrium is thus fully specified as

$$
\begin{aligned}
& x_{1}^{\ell}=\frac{2+\gamma(1-\lambda)}{6-2 \lambda+2 \gamma(1-\lambda)} a, \\
& x_{2}^{\ell}=\frac{2-\lambda}{6-2 \lambda+2 \gamma(1-\lambda)} a .
\end{aligned}
$$


It can be verified by tedious computation that $x_{1}^{\ell}$ exceeds the optimal activity level of $a / 4$, while $x_{2}^{\ell}$ is less than $a / 4$; the total activity $x_{1}^{\ell}+x_{2}^{\ell}$ is more than the efficient level of $a / 2$, so Leader-Follower social opinions lead to underuse of the commons in the aggregate.

Turning to comparative statics, when the leadership degree changes, we find that for all degree levels $\lambda$ and $\gamma$,

$$
\frac{\partial x_{1}^{\ell}}{\partial \lambda}=\frac{a}{2[3-\lambda+\gamma(1-\lambda)]^{2}}>0,
$$

and that for $\gamma<1$

$$
\frac{\partial x_{2}^{\ell}}{\partial \lambda}=\frac{a(\gamma-1)}{2[3-\lambda+\gamma(1-\lambda)]^{2}}<0 .
$$

The sum of these is positive for all $\lambda$ and $\gamma$ :

$$
\frac{\partial x_{1}^{\ell}}{\partial \lambda}+\frac{\partial x_{2}^{\ell}}{\partial \lambda}=\frac{a(1+\gamma)}{2[3-\lambda+\gamma(1-\lambda)]^{2}}>0
$$

When the degree of pessimism of the pessimist changes, we find that if $\lambda<1$,

$$
\frac{\partial x_{1}^{\ell}}{\partial \gamma}=\frac{a(1-\lambda)(1+\lambda \gamma-\lambda)}{2[3-\lambda+\gamma(1-\lambda)]^{2}}>0,
$$

and

$$
\frac{\partial x_{2}^{\ell}}{\partial \gamma}=\frac{a(2-\lambda)(\lambda-1)}{2[3-\lambda+\gamma(1-\lambda)]^{2}}<0 .
$$

The sum of these is negative:

$$
\frac{\partial x_{1}^{\ell}}{\partial \gamma}+\frac{\partial x_{2}^{\ell}}{\partial \gamma}=\frac{a(1-\lambda)(\lambda \gamma-1)}{2[3-\lambda+\gamma(1-\lambda)]^{2}}<0 .
$$

Our results are summarized below.

Theorem 3.1 Let $0<\lambda, \gamma<1$. In the Leadership Equilibrium applied to the given commons game, we have the following properties:

(i) In the resulting Leadership Equilibrium there is overuse of the commons.

(ii) Furthermore, an increase in the leadership degree of the larger player increases that player's activity level, reduces the smaller player's activity level, and increases the 
sum of their activity levels.

(iii) Finally, an increase in the follower degree of the smaller player increases the larger player's activity level, decreases the smaller player's activity level, and decreases the sum of their activity levels.

We emphasize that the main conclusion from the theorem stated is that leadership ambiguity has negative effects on the extraction of the commons. First, there is always overuse of the common pool resource under leadership ambiguity. Second, the more confident a negotiator is about her leadership position, the farther away from optimality the extraction from the common pool resource that results. 


\section{Some comparisons}

In the setting of our simple commons game we have considered three equilibrium concepts that are based on ambiguity considerations. In this section we discuss how these equilibrium concepts compare with regard to total extractions from the common resource. We show that there is no clear unambiguous ranking of these forms of ambiguity in terms of efficiency.

First, we compare the symmetric ambiguity equilibrium with the antipodal ambiguity equilibrium. Comparisons between the derived equilibria lead us to the conclusion that there are degree values for which the antipodal case is Pareto superior to the symmetric case and vice versa. The following statement makes that more precise.

Theorem 4.1 Let $0 \leqslant \lambda, \gamma \leqslant 1$ be two given degrees of optimism and pessimism. Consider the symmetric ambiguity equilibrium $x^{s}$ in $\Gamma^{s}(\lambda, \gamma)$ and the antipodal ambiguity equilibrium $x^{a}$ in the context of $\gamma^{a}(\lambda, \gamma)$. Then the following statements hold:

(i) If $\gamma<0.56 \lambda-0.01$, then $x_{1}^{s}+x_{2}^{s}>x_{1}^{a}+x_{2}^{a}$, i.e., the commons extraction under symmetric ambiguity is larger than the commons extraction under antipodal ambiguity.

(ii) If $\gamma>0.56 \lambda+0.01$, then $x_{1}^{s}+x_{2}^{s}<x_{1}^{a}+x_{2}^{a}$, i.e., the commons extraction under antipodal ambiguity is larger than the commons extraction under symmetric ambiguity.

The proof of Theorem 4.1 is illustrated in Figure 2.

Next we consider the leadership equilibrium concept in comparison with the symmetric ambiguity equilibrium. Again we compare the total extraction from the commons under these two different regimes. Computations show that two situations emerge:

Theorem 4.2 Let $0 \leqslant \lambda, \gamma \leqslant 1$ be two given degrees of optimism and pessimism. Consider the symmetric ambiguity equilibrium $x^{s}$ in $\Gamma^{s}(\lambda, \gamma)$ and the leadership equilibrium $x^{\ell}$ in the context of $\gamma^{\ell}(\lambda, \gamma)$. Then the following statements hold:

(i) If $\gamma<0.4 \lambda-0.01$, then $x_{1}^{s}+x_{2}^{s}>x_{1}^{\ell}+x_{2}^{\ell}$, i.e., the commons extraction under symmetric ambiguity is strictly larger than the commons extraction under the leadership equilibrium.

(ii) If $\gamma>0.4 \lambda+0.01$, then $x_{1}^{s}+x_{2}^{s}<x_{1}^{\ell}+x_{2}^{\ell}$, i.e., the commons extraction under symmetric ambiguity is strictly smaller than the commons extraction under the leadership equilibrium. 


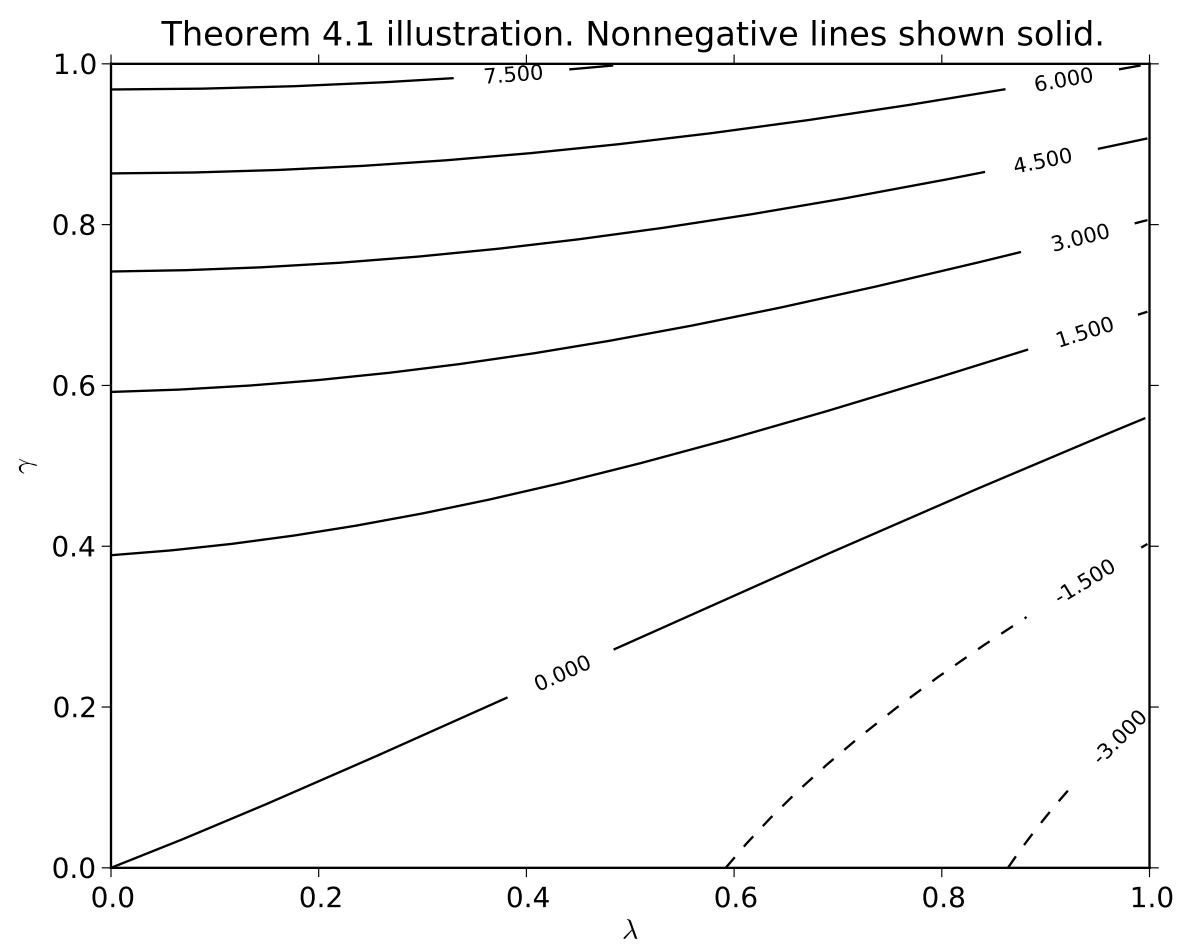

Figure 2: Illustration of Theorem 4.1. Several level sets of $x_{1}^{a}+x_{2}^{a}-x_{1}^{s}-x_{2}^{s}$ are shown.

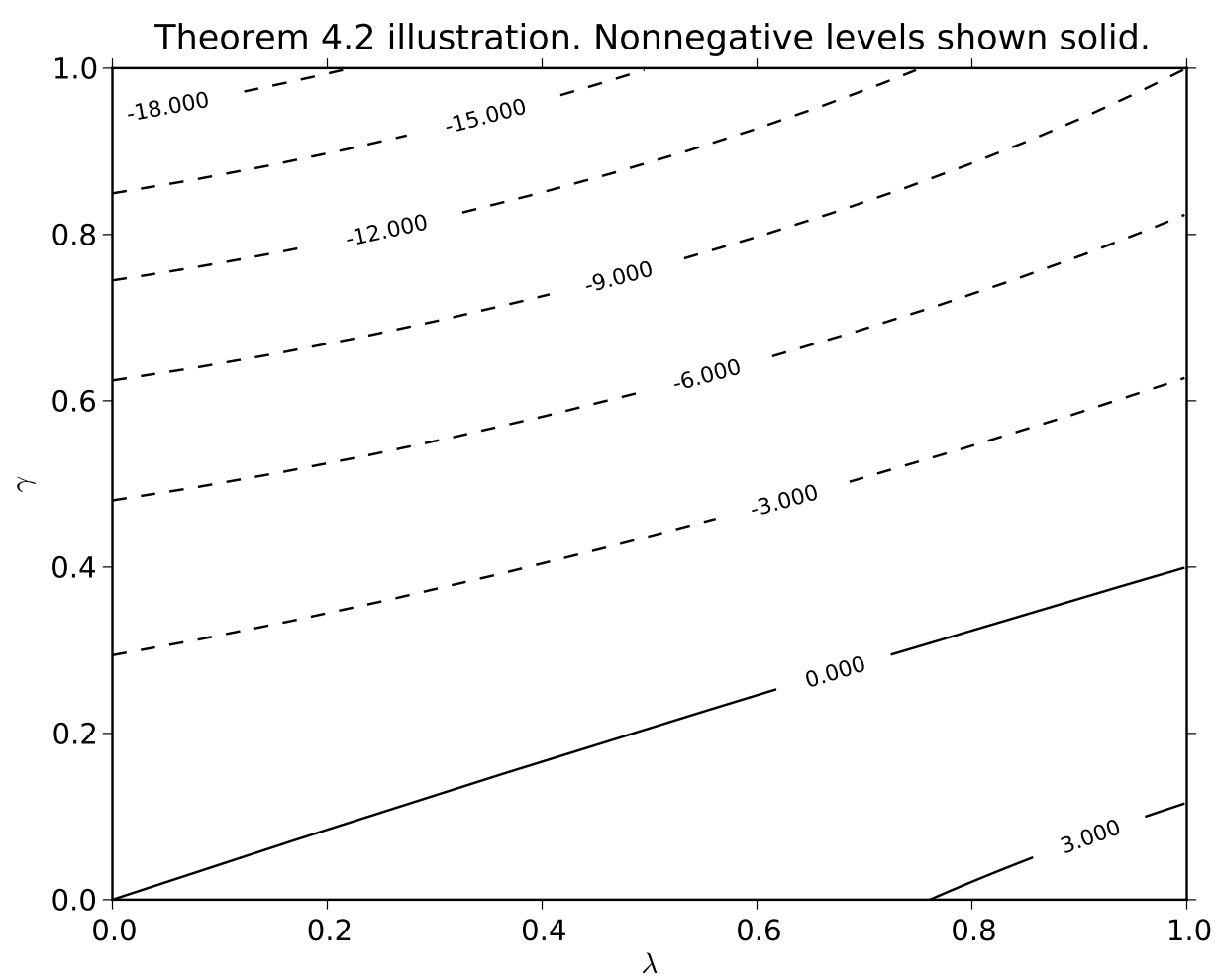

Figure 3: Illustration of Theorem 4.2. Several level sets of $x_{1}^{s}+x_{2}^{s}-x_{1}^{\ell}-x_{2}^{\ell}$ are shown. 


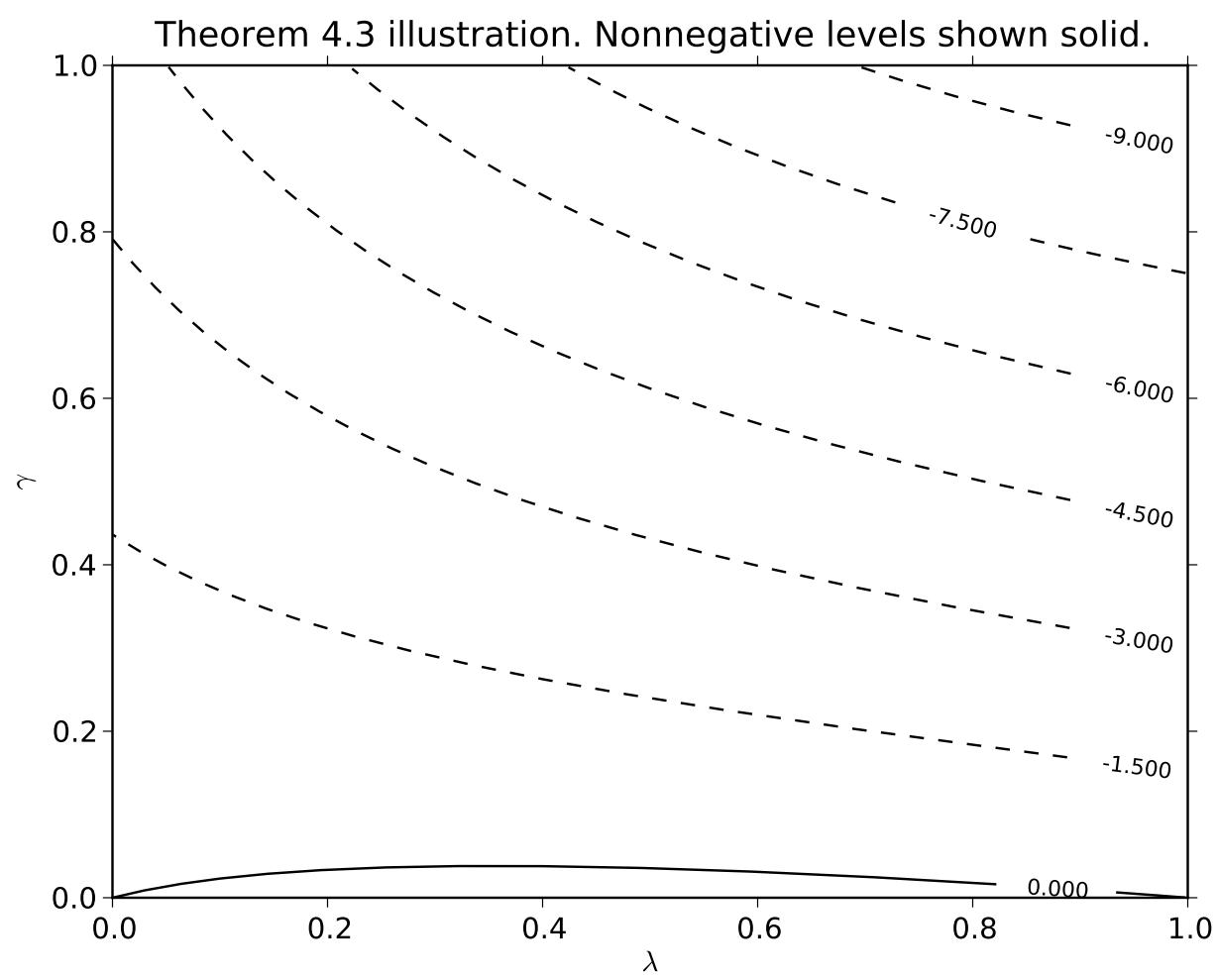

Figure 4: Illustration of Theorem 4.3. Several level sets of $x_{1}^{a}+x_{2}^{a}-x_{1}^{\ell}-x_{2}^{\ell}$ are shown.

The proof of Theorem 4.2 is illustrated in Figure 3.

The comparison of the leadership equilibrium and the case of antipodal ambiguity is less straightforward. Again there are different sets of degree values for which opposite comparisons hold. For example, for $\gamma=0$ and $\lambda=\frac{1}{2}$ we have that the total extraction of the commons under antipodal ambiguity is larger than the total extraction under the leadership equilibrium, representing structural ambiguity.

On the other hand, for most degree values the opposite holds as is stated in the following assertion:

Theorem 4.3 Let $0 \leqslant \lambda, \gamma \leqslant 1$ be two given degrees of optimism and pessimism. Consider the antipodal ambiguity equilibrium $x^{a}$ in $\Gamma^{a}(\lambda, \gamma)$ and the leadership equilibrium $x^{\ell}$ in the context of $\gamma^{\ell}(\lambda, \gamma)$.

If $\gamma \geqslant 0.075$, it holds that $x_{1}^{a}+x_{2}^{a}<x_{1}^{\ell}+x_{2}^{\ell}$, i.e., the commons extraction under antipodal ambiguity is strictly smaller than the commons extraction under the leadership equilibrium.

Finally, the proof of Theorem 4.3 is illustrated in Figure 4.

In other words, if the degree of pessimism under antipodal ambiguity and the follower degree for the case of structural ambiguity is sufficiently large, structural ambiguity results into higher total extraction from the commons than for the case of antipodal ambiguity. 


\section{Coda}

We have considered a standard game-theoretic model of the tragedy of the commons to illustrate the global environmental situation. We solved the model by means of ambiguity equilibrium and a modification of ambiguity equilibrium intended to open the possibility of capturing in the model socially determined optimistic and pessimistic attitudes of the players based on leadership considerations. While very simple, the model yields intriguing results that appear to capture the general outlines of the behavior of developed and developing countries over the past several decades.

Even more intriguing are the new questions that arise from the viewpoint we have adopted regarding the extension of ambiguity equilibrium to include social determination of the components of the model; that is, (i) of the most selfish action a player can be considered to take, and (ii) of the player's degree of optimism and pessimism. To do this, we have departed from the original ambiguity equilibrium concept and therefore cannot point to its axiomatic foundation for conceptual support. However, we believe there is an intuitive appeal to our analytical departure. As for the new questions it creates, we find these the most interesting: (i) to formulate a laboratory experiment to test the performance of our extension of ambiguity equilibrium, (ii) to embed our extension into an explicit model of social belief formation, and (iii) to model an enriched dynamic model of negotiations. We consider these questions very promising for future research on this important subject.

\section{References}

Barrett, S. (2003): Environment $\mathcal{E}$ Statecraft. Oxford University Press, Oxford, UK.

Camerer, C., and M. Weber (1992): "Recent Developments in Modelling Preferences: Uncertainty and Ambiguity," Journal of Risk and Uncertainty, 5, 325-370.

Chateauneuf, A., J. Eichberger, and S. Grant (2007): “Choice under Uncertainty with the Best and Worst in Mind: Neo-additive Capacities," Journal of Economic Theory, 137, 538567.

Choquet, G. (1954): “Theory of Capacities,” Annales de l'Institute Fourier, 5, 131-295.

Eichberger, J., AND D. Kelsey (2000): "Non-Additive Beliefs and Strategic Equilibria," Games and Economic Behavior, 30, 183-215. 
(2006): "Optimism and Pessimism in Games," Working Paper, University of Heidelberg, Germany.

_ (2007): "Are the Treasures of Game Theory Ambiguous?," Working Paper, University of Heidelberg, Germany.

Eichberger, J., D. Kelsey, and B. C. Schipper (2006): “Granny versus Game Theorist: Ambiguity in Experimental Games," Working Paper, UC Davis.

_ (2007): “Ambiguity and Social Interaction,” Working Paper, University of Heidelberg, Germany.

Ellsberg, D. (1961): "Risk, Ambiguity and the Savage Axioms," Quarterly Journal of Economics, 75, 643-669.

Falk, A., E. Fehr, and U. Fischbacher (2001): "Appropriating the Commons: A Theoretical Explanation," CESifo working paper No. 474.

KnIGHT, F. (1921): Risk, Uncertainty and Profit. Houghton Mifflin, New York, NY.

Kolstad, C. D. (2007): "Systematic Uncertainty in Self-Enforcing International Environmental Agreements," Journal of Environmental Economics and Management, 53, 68-79.

Savage, L. J. (1954): Foundations of Statistics. Wiley \& Sons, New York, NY.

SchmeIder, D. (1989): "Subjective Probability and Expected Utility Without Additivity," Econometrica, 57, 571-587.

UlPh, A. M. (2004): "Stable International Environmental Agreements with a Stock Pollutant, Uncertainty and Learning," Journal of Risk and Uncertainty, 29, 53-73. 